\title{
Numerical and Experimental Investigation on Braking Characteristics of an Electro-Rheological (ER) Micro Brake*
}

\author{
Jinghui PENG ${ }^{* *}$, Takanori TOGAWA ${ }^{* *}$, Takuma TACHIBANA ${ }^{* *}$, Yutaka TANAKA ${ }^{* *}$ \\ Micromouse contest is a kind of world popular competition for micro-mobile robots. Due to excellent mechanical \\ performance, the electro-rheological (ER) brake has a great potential to realize smooth and rapid turning of the \\ micromouse in the maze. A small-scale disc type ER brake is proposed and developed in this paper. Aiming to predict the \\ performance of the ER brake, the mathematical model of the braking torque is established based on the constitutive \\ model of one particle type ER fluid (ERF). The static and dynamic braking characteristics of the ER brake under different \\ applied voltages are simulated and tested. The braking effect is verified by the good agreement between the simulated and \\ tested results.
}

Keywords: Soft brake, Electro-rheological fluid, Mathematical model, Braking test, Micromouse

\section{Introduction}

As the combination of both mechanical and electronic systems, micro mechatronics is attracting more and more research interests with the significant development of micromachining techniques ${ }^{1)}$. The micro mechatronics system is expected to be the most potential high-tech integrated product, especially in electronic and automotive industries.

Micromouse contest is a competition for micro mechatronics to compete for speed and intelligence, which is especially popular in UK, U.S., Japan, Singapore and so on. The micromouse is a kind of maze-solving autonomous micro wheeled robot. The maze is usually made up of $16 \times 16$ square cells, with each cell $18 \times 18 \times 50 \mathrm{~cm}$ in length, width, and height. In the contest, the micromouse must find its way from the starting position to the destination in the shortest time by mapping and optimizing the best path intelligently ${ }^{2}$. How to turn smoothly and rapidly, and to miniaturize its components have become great challenges in the development of micromouse ${ }^{3)}$.

As a soft brake, the ER braking device has outstanding mechanical performance such as large output dynamics, fast speeds of response and continuous adjustment ${ }^{4}$. The viscosity and yield stress of the ERF can be controlled widely and rapidly by changing the applied electric field ${ }^{5}$. Then large damping forces can be generated and adjusted to resist the motion of the micro mechatronic devices. As a

\footnotetext{
Manuscript received January 31, 2018

** Hosei University

(Fujimi 2-17-1 Chiyoda-ku Tokyo, Japan)

E-mail: y_tanaka@hosei.ac.jp
}

promising application, the development of small-scale ER braking device has a great potential to perfectly solve the problems met by the micro-mobile robot.

In this paper, a small scale disk type ER brake for micromouse is proposed and developed. To achieve the design goals of smaller scale, faster response speed and lower applied voltage, the braking characteristics of the ER brake are simulated and tested. The simulation and experimental results are compared and analyzed thoroughly.

\section{Nomenclature}

$\begin{array}{clll}d_{1} & : & \text { effective outer diameter } \\ d_{2} & : & \text { effective inner diameter } \\ E & : & \text { strength of the electric field } \\ h & : & \text { electrode gap } \\ J & : & \text { total moment of inertia } \\ K_{p} & : & \text { ratio of load torque and angular speed } \\ r & : & \text { radial coordinate } \\ T_{B} & : & \text { braking torque } \\ T_{f} & : & \text { friction torque } \\ T_{m} & : & \text { driving torque } \\ \dot{\gamma} & : & \text { shear rate } \\ \mu_{0} & : & \text { original viscosity } \\ \tau & : & \text { shear stress } \\ \tau_{0} & : & \text { dynamic yield stress } \\ \omega & : & \text { angular speed } \\ \omega_{0} & : & \text { angular speed without load }\end{array}$

\section{Structure and Working Principle}

The schematic diagram of the disk type ER brake designed in this paper is presented in Fig. 1. It mainly consists of four parts: the rotational shaft, the electrodes, the bearings and the case. The rotational shaft is directly 
connected to the DC motor. The electrodes include one positive electrode and two negative electrodes, which form two effective braking layers. The miniature bearing on the right side is a support for shaft, while the sealed bearing on the left side can provide a sealed chamber for ERF.

During the working process, the positive electrode fixed on the rotational shaft rotates with the shaft, while the negative electrodes stay still with the case. Once high voltage is applied between the positive and negative electrodes, the apparent viscosity of the ERF in the braking layers increases in milliseconds. Large braking torques are generated and applied on both sides of the positive electrode due to the solidified ERF. Then the DC motor can be halted by the ER brake rapidly. When the voltage is removed, the initial viscosity of the ERF is resumed and the speed of the DC motor is recovered quickly. Besides, it is possible to restrict the rotational speed automatically due to the controllability of the apparent viscosity of ERF. The response time of the rotational shaft can also be adjusted by the applied voltage.

In this design, the positive electrode is electrified by power source through the conductive bearings and the shaft. While, the negative electrodes are electrified through the wire welded to the surface of the left negative electrode. Copper spacers are placed between the negative electrodes and the case for conduction and localization.

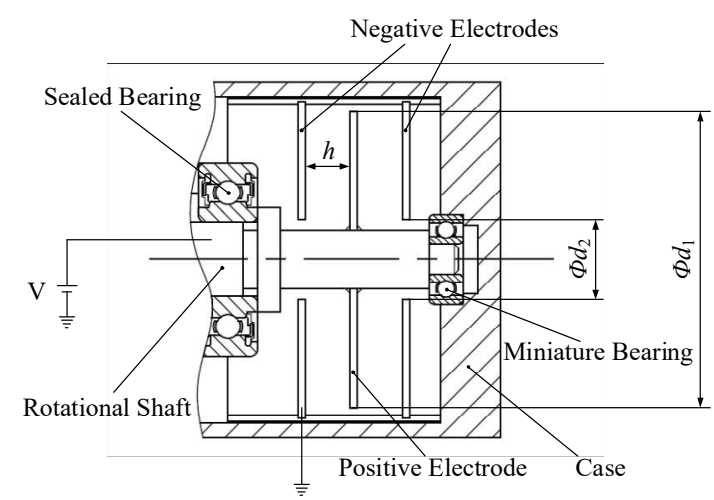

Fig.1 Construction of the ER brake

\section{Numerical Model}

\subsection{Constitutive Model for Particle Type ERF}

As a kind of representative ERF, the rheological property of particle type ERF is shown in Fig. 2. It can be seen that the particle type ERF behaves as non-Newtonian fluid under the external electric field, while it behaves as Newtonian fluid when the electric field is removed. Thus, the constitutive characteristics of particle type ERF can be described using the Bingham model, shown as below.

$$
\tau=\tau_{0}+\mu_{0} \dot{\gamma}
$$

The particle type ERF used in this work is manufactured by the ER Tec Co., Ltd, Japan. According to the property data provided by the manufacturer, the parameters in Eq. (1) can be identified by adapting least square method, as follows:

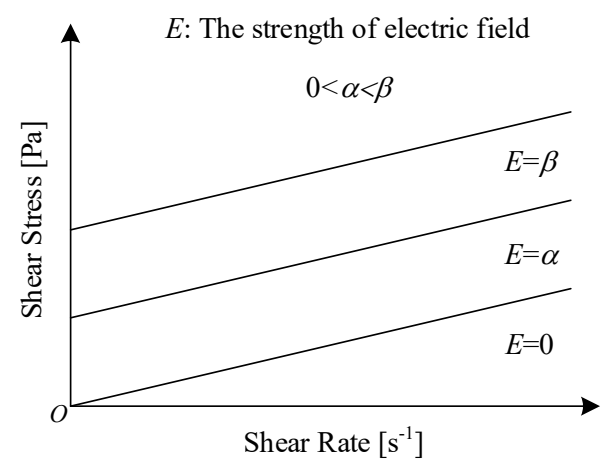

Fig.2 Rheological properties of particle type ERF

$$
\begin{aligned}
& \tau_{0}=317.5 E^{1.834} \\
& \mu_{0}=0.225
\end{aligned}
$$

where the units of $E$ and $\mu_{0}$ are $\mathrm{kV} / \mathrm{mm}$ and $\mathrm{Pa} \cdot \mathrm{s}$, respectively.

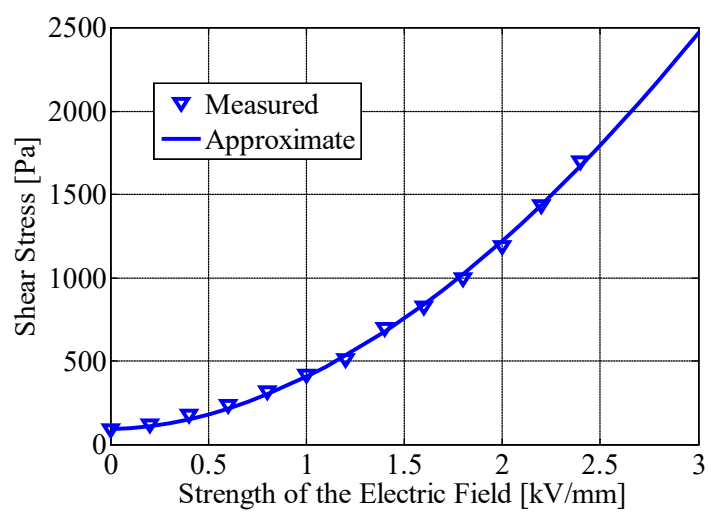

Fig.3 Shear stress vs. strength of the electric field for particle type

$$
\text { ERF at } \dot{\gamma}=400 \mathrm{~s}^{-1}
$$

The rheological properties of the ERF are obtained with coaxial cylinder viscometer. Figure 3 illustrates the relationship between the shear stress and the strength of electric field for the particle type ERF under the shear rate of $400 \mathrm{~s}^{-1}$. It can be seen that the measured and approximate constitutive curves achieve a good agreement. Besides, we can see that the shear stress of the particle type ERF 
increases with power-law of the strength of electric field. This conclusion is consistent with the results of the previous studies $^{6)-7)}$.

\subsection{The Mathematical Model of the ER Braking Torque}

To investigate the braking characteristics of the ER brake, it is necessary to establish the mathematical model of the ER braking torque. According to the working principle of the DC motor, the torque equilibrium equation of the ER brake can be written as

$$
T_{B}=T_{m}-T_{f}-J \dot{\omega}=K_{p}\left(\omega_{0}-\omega\right)-J \dot{\omega}
$$

Based on the constitutive characteristics of ERF, the braking torque on the positive electrode of particle type ER brake can be obtained through surface integral as

$$
T_{B}=\int_{\frac{d_{2}}{2}}^{\frac{d_{1}}{2}} 4 \pi r^{2} \tau d r
$$

Plug Eq. (1) into Eq. (5), we can get

$$
T_{B}=\int_{\frac{d_{2}}{2}}^{\frac{d_{1}}{2}} 4 \pi r^{2}\left(\tau_{0}+\mu_{0} \dot{\gamma}\right) d r
$$

Due to the high viscosity of the ERF and the small electrode gaps, the Bingham flow inside the braking layers is generally assumed to be laminar flow. Thus, the shear rate of the ERF can be written as

$$
\dot{\gamma}=\frac{r \omega}{h}
$$

Then, Eq. (6) can be deduced to be

$$
T_{B}=\frac{\pi \tau_{0}}{6}\left(d_{1}^{3}-d_{2}^{3}\right)+\frac{\pi \mu_{0} \omega}{16 h}\left(d_{1}^{4}-d_{2}^{4}\right)
$$

Based on Eq. (4) and Eq. (8), the static characteristics of the ER brake can be described as

$$
\omega=\frac{K_{p} \omega_{0}-\frac{\pi \tau_{0}}{6}\left(d_{1}^{3}-d_{2}^{3}\right)}{K_{p}+\frac{\pi \mu_{0}}{16 h}\left(d_{1}^{4}-d_{2}^{4}\right)}
$$

The dynamic characteristics of the ER brake can be obtained using Simulink software. The established Simulink model for particle type ER brake is shown in Fig. 4. The switch blocks are used to simulate different working conditions of the DC motor. The electrical time constant of the ER brake is assumed to be $5 \mathrm{~ms}$, while the one of the DC motor can be neglected.

\section{Experimentation}

To test the real braking characteristics, a prototype of the ER brake is fabricated using $3 \mathrm{D}$ printer. Figure 5 shows the exploded view of the tested brake. The DC motor (1717T006SR, Faulhaber, Germany) used in the experiment is designated by the micromouse contest, which has a stall torque of $5.34 \mathrm{mN} \cdot \mathrm{m}$ and a no-load speed of $14000 \mathrm{~min}^{-1}$. Considering the specification and size of the DC motor and micromouse, the initial design dimensions of the ER brake are as follows: the gap between the opposite electrodes is 3 $\mathrm{mm}$, and the effective outer and inner diameters of the electrodes are $15 \mathrm{~mm}$ and $5.4 \mathrm{~mm}$, respectively. Then, $J$ and $K_{p}$ in Eq. (4) can be calculated to be $8.53 \times 10^{-8} \mathrm{~kg} \cdot \mathrm{m}^{2}$ and $3.64 \times 10^{-6} \mathrm{~N} \cdot \mathrm{m} /(\mathrm{rad} / \mathrm{s})$, respectively.

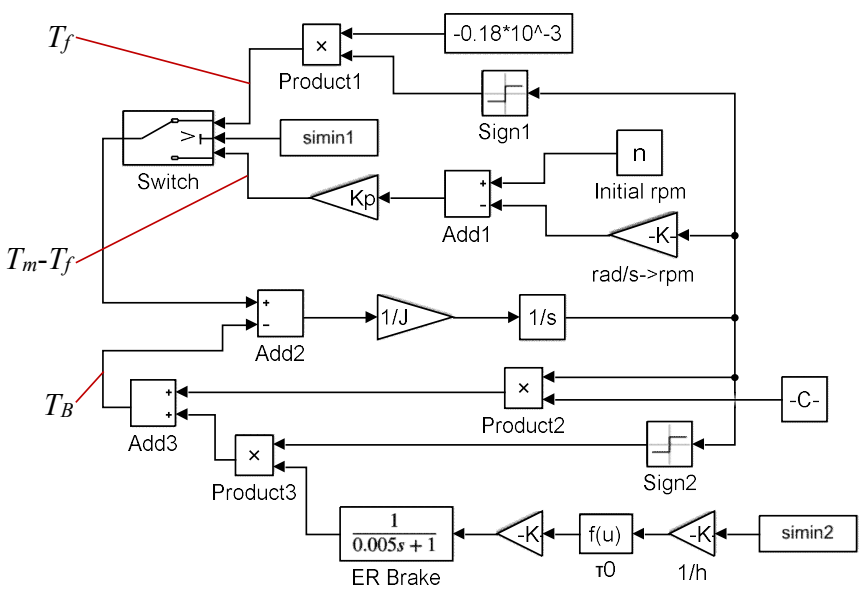

Fig.4 Simulink model of particle type ER brake

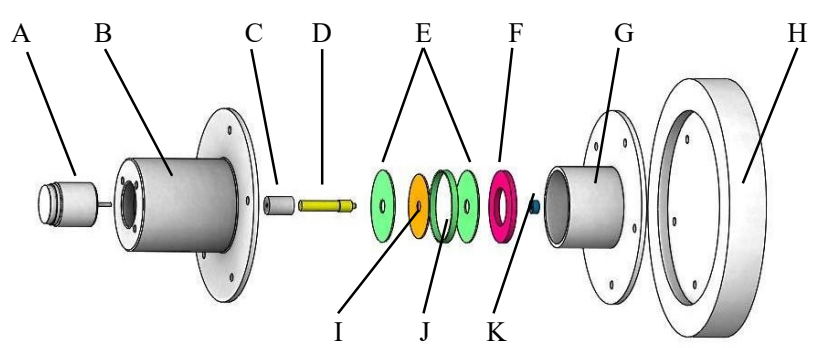

Fig. 5 Exploded view of the ER brake prototype. (A) DC motor, (B and $\mathrm{G}$ ) case, (C) connector, (D) conductive shaft, (E) negative electrode, $(\mathrm{F})$ supporting spacer, $(\mathrm{H})$ baseplate, (I) positive electrode,

$(\mathrm{J})$ conductive spacer and $(\mathrm{K})$ bearing

The composition of the experimental system to measure the performance of the ER brake is shown in Fig. 6. The ER brake is driven by the DC motor directly. The power supplies of the DC motor and the ER brake are controlled through the micro-computer (RX621, Renesas Electronics Corporation, Japan). The rotational speed of the DC motor is measured by the magnetic encoder (IE2-512, Faulhaber, Germany) integrated in the DC motor. The encoder pulses are counted by the micro-computer at the phase counting mode. Then, the counting data is sent to the PC through 
serial communication by an interface switching chip (FT232RL, IC Lando Inc., Japan). The counting data is transferred to angular speed and the speed responses of the DC motor are acquired finally. The braking characteristics of the ER brake under different working conditions are tested in this experiment.

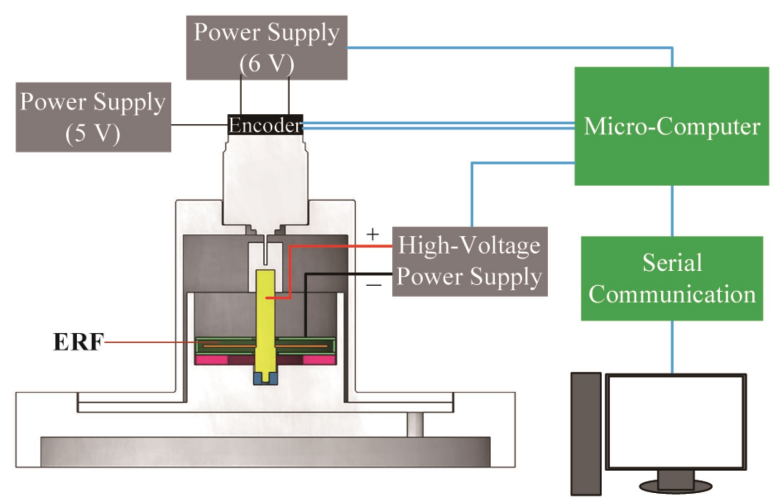

Fig.6 Experimental set-up for braking test

\section{Results and Discussion}

\subsection{Simulation results}

Based on the established mathematical model, the braking characteristics of the ER brake prototype can be calculated. First, the static characteristics are examined and the simulated rotational speed of the DC motor and the braking torque changing with the applied voltage are shown in Fig. 7.

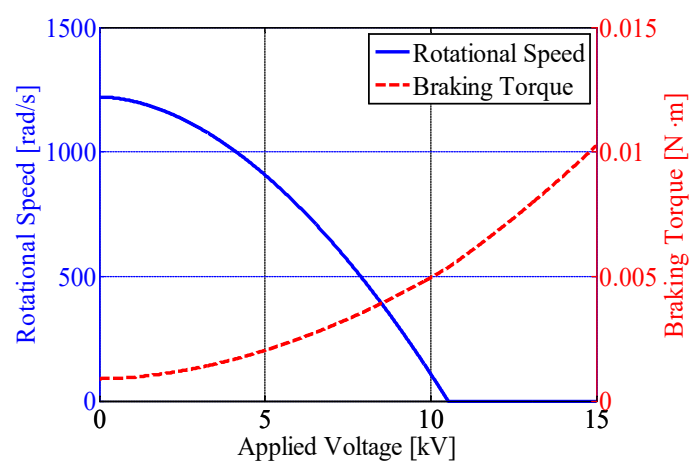

Fig.7 Simulated rotational speed and braking torque vs. applied

$$
\text { voltage }
$$

From Fig. 7, it can be seen that the rotational speed decreases with power-law of the applied voltage and the stopping voltage is about $10.52 \mathrm{kV}$, while the braking torque increases with the applied voltage. When the applied voltage is higher than $10.52 \mathrm{kV}$, the braking torque is beyond the maximum output torque of the DC motor. Then the motor is halted. Besides, when the applied voltage is $0 \mathrm{~V}$, the rotational speed is decreased from $1466 \mathrm{rad} / \mathrm{s}$ to $1221 \mathrm{rad} / \mathrm{s}$ due to the initial viscosity of the ERF. Here, $1466 \mathrm{rad} / \mathrm{s}$ is the no-load rotational speed of the DC motor. Thus, ERF with the lower initial viscosity should be selected to avoid energy loss in the running stage after the braking requirement is met.

The dynamic characteristics of the ER brake can be obtained by the numerical simulation using Simulink software. The simulated rotational speeds of the DC motor under different applied voltages are shown in Fig. 8.

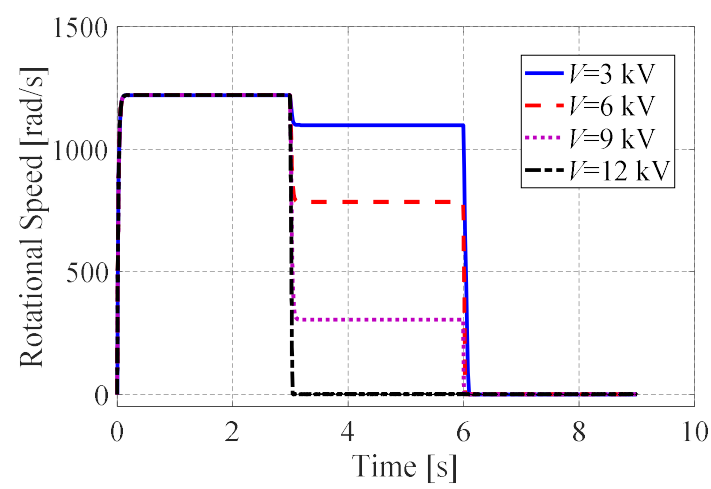

(a) Whole time histories

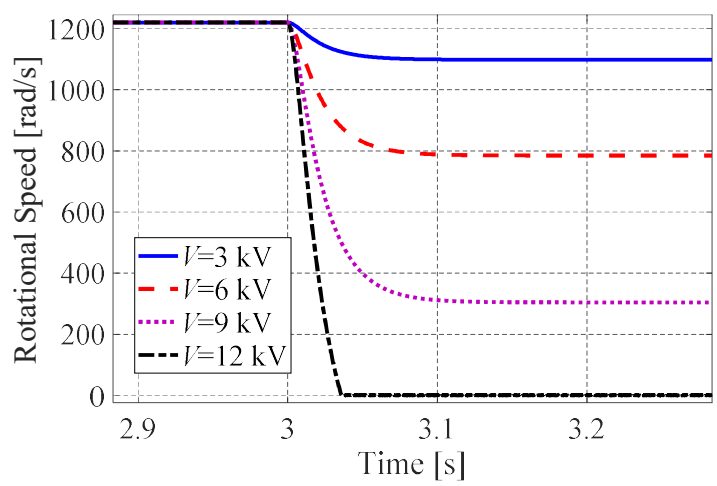

(b) Part time histories

Fig. 8 Simulated rotational speed change of the DC motor

In Fig. 8, the high voltage is applied from $3 \mathrm{~s}$ to $9 \mathrm{~s}$, while the ERF works at the initial viscosity for the rest of the time. The DC motor is powered on from $0 \mathrm{~s}$ to $6 \mathrm{~s}$. It is obvious that the rotational speed decreases faster to a lower level when higher voltage is applied because of the exerted larger braking torque.

In the case where the micromouse need to be halted completely and rapidly, the DC motor should be powered off at the same time. When the applied voltage is $10.52 \mathrm{kV}$, the comparison of the braking characteristics between two working conditions where the DC motor is powered on or powered off in the braking stage are conducted. The numerical simulated results are shown in Fig. 9.

In Fig. 9, the condition 1 means the DC motor is always powered on, while the condition 2 means the DC motor is 
powered off in the braking stage. It can be seen that the braking response time of condition 1 and 2 are $101 \mathrm{~ms}$ and $22 \mathrm{~ms}$, respectively. When the DC motor is powered off in the braking stage, the braking response time of the particle type ER brake is shortened greatly.

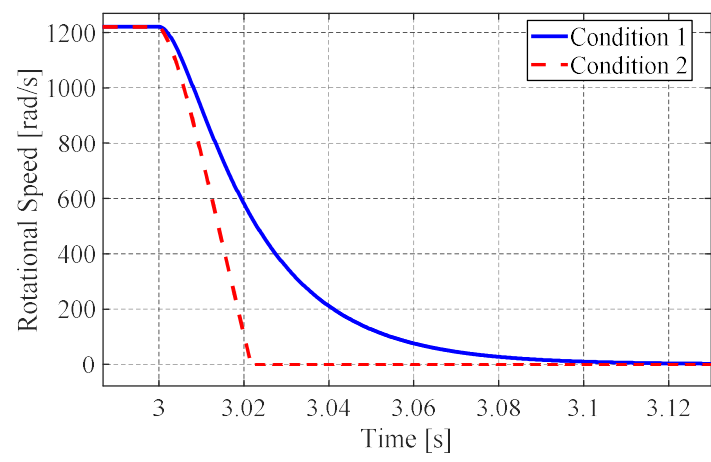

Fig.9 Comparison of the rotational speed change under different working conditions

\subsection{Experimental results}

To obtain the real braking performance of the ER brake and verify the effectiveness of the mathematical model, the rotational speeds of the DC motor under different applied voltages are measured. Because the minimum distance between the negative electrode and the rotational shaft is only $0.7 \mathrm{~mm}$, high voltage breakdown phenomenon is likely to happen when the applied voltage is higher than $2 \mathrm{kV}$. Thus, in this experiment, the braking characteristics of the ER brake are tested under the applied voltage lower than 2 $\mathrm{kV}$.

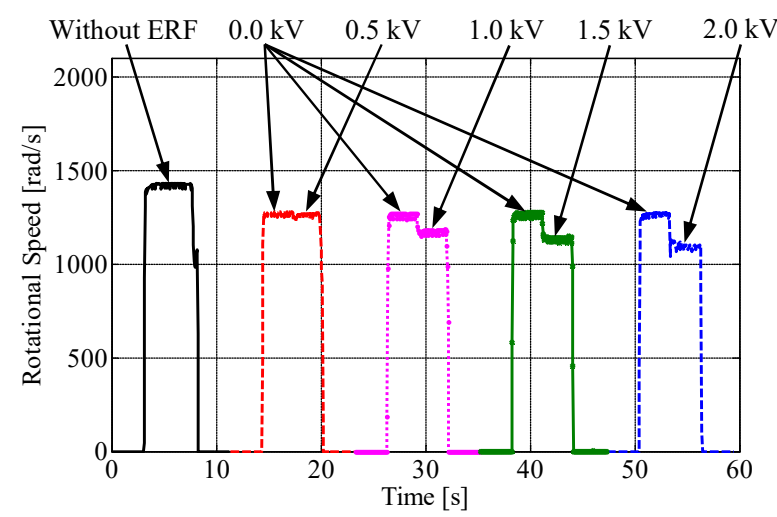

Fig.10 Tested rotational speed change of the DC motor

The tested rotational speed of the DC motor is shown in Fig. 10. The experimental results indicate that the static rotational speed decrease with the increasing applied voltage, which is consistent with the simulation results. After acquiring the static speed, the static braking torques under different applied voltages can be estimated by Eq. (4). The tested rotational speeds and braking torques at the static state are shown in Fig. 11. The changing trends of the speed and braking torque with applied voltage agree well with the simulation results. When the applied voltage is $2 \mathrm{kV}$, the estimated braking torque is about $1.327 \mathrm{mN} \cdot \mathrm{m}$ which is not enough to halt the DC motor. The braking response time is around $150 \mathrm{~ms}$ when the DC motor is powered off under the voltage of $2 \mathrm{kV}$.

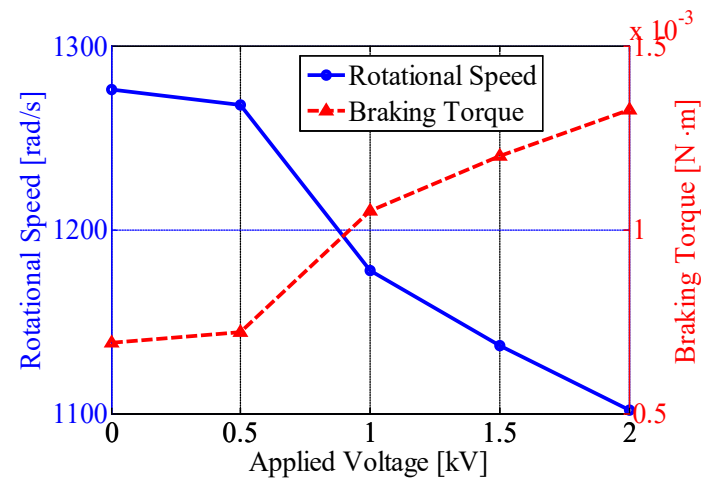

Fig.11 Tested rotational speed and braking torque vs applied voltage

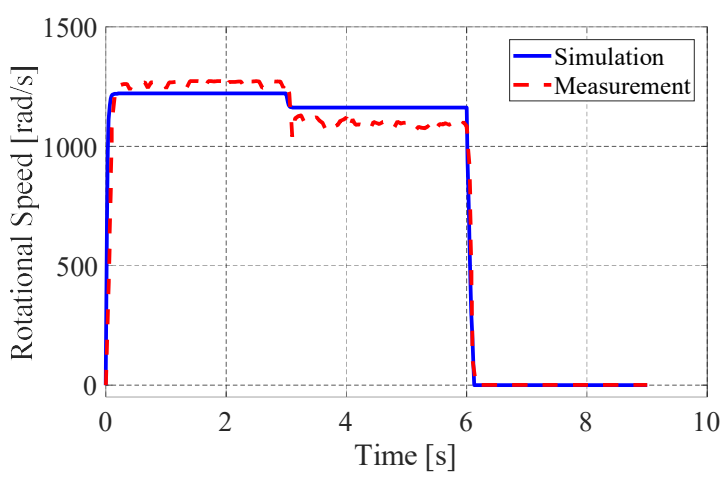

Fig.12 The comparison of simulated and tested rotational speed change

\subsection{Comparison between numerical and experimental results}

Detailed comparison of the calculated and tested rotational speed change is carried out when the applied voltage is $2 \mathrm{kV}$, as shown in Fig. 12. It can be seen that the simulation and experimental results achieve a good agreement. Both results indicate an obvious speed reduction when the high voltage is applied. The simulated and tested braking response times are around $127 \mathrm{~ms}$ and $150 \mathrm{~ms}$, respectively.

The comparison of the simulated and tested static rotational speeds of the DC motor is listed in Table. 1. It can be seen that the errors between the simulation and the experiment are below $6 \%$. The errors are mainly caused by the deviation between the real viscosity property of the ERF and the provided property data by the manufacturer. Further 
Table 1 The simulated and tested rotational speed of the DC motor

\begin{tabular}{|c|c|c|c|c|c|}
\hline & $0.0[\mathrm{kV}]$ & $0.5[\mathrm{kV}]$ & $1.0[\mathrm{kV}]$ & $1.5[\mathrm{kV}]$ & $2.0[\mathrm{kV}]$ \\
\hline Simulated [rad/s] & 1221 & 1216 & 1204 & 1186 & 1163 \\
\hline Tested $[\mathrm{rad} / \mathrm{s}]$ & 1276 & 1268 & 1178 & 1137 & 1102 \\
\hline Error $[\%]$ & 4.31 & 4.10 & 2.21 & 4.31 & 5.54 \\
\hline
\end{tabular}

viscosity measurement need to be carried out to obtain more accurate constitutive model of the ERF used in our work.

\section{Conclusion}

To examine the performance of a disc type small scale ER brake for micromouse, the braking characteristics are studied through numerical and experimental analysis. The rotational speed and braking torque responses are obtained and compared in detail. Both simulation and experimental results verify the braking effect of the ER brake. This study can establish a fundamental knowledge for the application of the small scale ER brake in micro mechatronics systems.

However, our work still has some limitations. In the future, more efforts need to be done to solve the high voltage breakdown problems for the acquirement of the braking performances under higher voltages. In addition, the shear stress distribution on the positive electrode needs to be studied to obtain more accurate braking torque responses.

\section{Acknowledgments}

This research is supported by the MEXT-Supported Program for the Strategic Research Foundation at Private Universities, Japan, 'Next-generation energy conversion systems sustaining green technology, Research Center for Micro-Nano Technology, Hosei Univeristy.' This work was supported by Adaptable and Seamless Technology Transfer Program through Target-driven R\&D (A-Step), Japan Science and Technology Agency, Grant Number AS251Z00941K.

\section{References}

1) Ishihara, H., Arai, F., Fukuda, T.: Micro Mechatronics and Micro Actuators, Mechatronics, IEEE/ASME Transactions on, Vol.1, No.1, p.68-79 (1996)

2) Tanaka, Y., Yokota, S.: Design and Fabrication of Micro Pump for Functional Fluid Power Actuation System, Next-Generation Actuators Leading Breakthroughs, Springer London, p.153-164 (2010)

3) Fan, X., Sakama, S., Togawa, T., Tanaka, Y.: Design and Fabrication of ER Braking Device for Micromouse, Proc. 7th Int. Conf. on Fluid Power and Mechatronics (FPM2015), IEEE, p.729-733 (2015)

4) Yoshida, K., Kamiyama, K., Kim, J. W., Yokota, S.: An Intelligent Microactuator Robust Against Disturbance Using Electro-Rheological Fluid, Sensors and Actuators A: Physical, Vol.175, p.101-107 (2012)

5) Tan, K. P., Stanway, R., Bullough, W. A.: Braking Responses of Inertia/Load by Using an ElectroRheological (ER) Brake, Mechatronics, Vol.17, No.6, p.277-289 (2007)

6) Wu, C. W., Conrad, H.: Dielectric and Conduction Effects in Ohmic Electrorheological Fluids, Journal of Physics D: Applied Physics, Vol.30, No.18, p.2634-2642 (1997)

7) Morishita, S.: Applications of Electrorheological Fluid and Its Feasibility, The Institute of Electronics, Information, and Communication Engineers, Vol.57, No.318, p.57-62 (1996) 